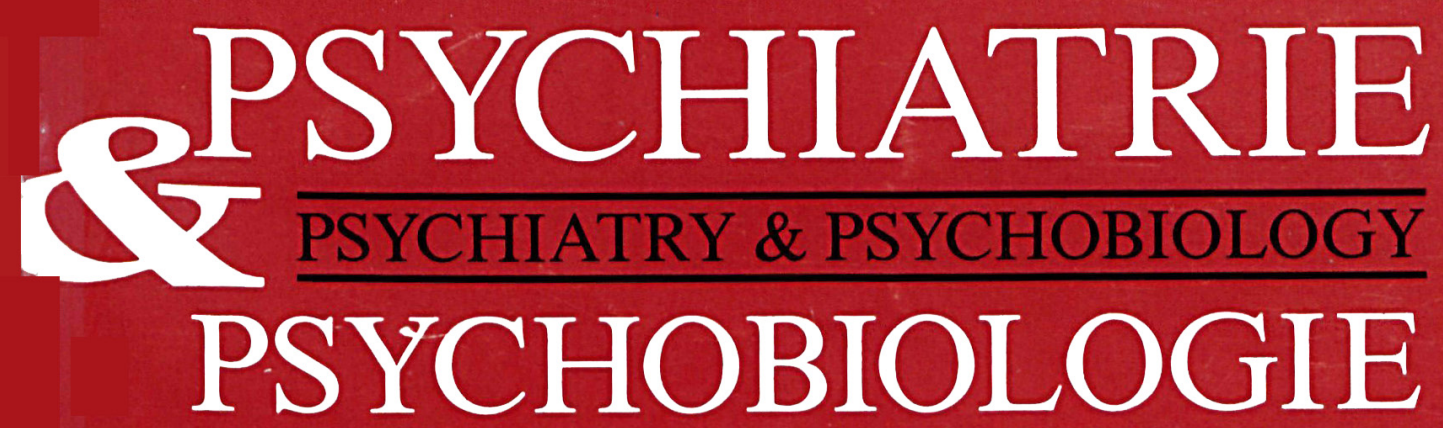

VOL. 4/No. 1

ISSN 0767-399 X

SCSPED II (1) $1-80$

PSYPEK (4) (1)

Rédacteurs en chef: P. Boyer, Paris/Sainte-Anne, J.D. Guelfi, Paris/Sainte-Anne, Y. Lfcrubier, Paris/Salpêtrièrc

Rédacteurs associés

Psychopathologie

J. AdEs, Paris/Colombes

H.S. Akiskal, Memphis, TN

J. Dalery, Lyon

R. Klein-Gittelman, New York

H. Katschnig, Vienne

R. Levy, Londres

H.G. POPE, Belmont, MA

W. REIN, Tübingen

Diagnostic et classification

N. Mombour, Munich

C.B. Pull, Luxembourg

N. Sartorius, Genève

R.L. Spitzer, New York

Psychobiologie

M. ACKenHeIL, Munich
P. Baumann, Lausanne

J.-P. Boulenger, Caen

D. Kemali, Naples

P. Lemoine, Lyon/Bron

\section{Psychothérapie}

A. Braconnier, Paris

S. CONSOLI, Paris

P. Jeammet, Paris

C. Perris, Ümea

I. Marks, Londres

\section{Chimiothérapie}

G. Cassano, Pise

J.A. Costa E Silva, Rio de Janeiro

D. Ginestet, Paris/Villejuif

G. Sedvall, Stockholm

R. Takahashi, Tokyo $†$

A. Villeneuve, Beaufort/Québec
Psychopharmacologie clinique

J.M. Danion, Strasbourg

S.Z. Langer, Paris

A.J. PUECH, Paris

T.W. Roвbins, Cambridge, U.K.

Comportement animal et pharmacologie

J. Costentin, Rouen

$\mathrm{Ph}$. Soubrie, Montpellier

Neuropsychologie

M. de BonIs, Paris/Kremlin-Bicêtre

J.A. Gray, Londres

Epidémiologie et Analyse des données

J. Fermanian, Paris

R. von FRENCKELL, Liège

J. Lellouch, Paris/Villejuif

J.P. Lépine, Paris

F. Roullion, Paris/Colombes

\title{
Comité Scientifique
}

N.C. Andreasen, lowa City, IO

M. Asberg, Stockholm

J.L. Ayuso, Madrid

T.A. BAN, Nashville, TN

P. BERner, Vienne

D. Bовоn, Liège

M. Bourgeols, Bordeaux

M. CaSAS, Barcelone

J. Cottraux, Lyon

R. Dantzer, Bordeaux

MENDLEWICZ, Bruxelles Peliet, Saint-Etienne Pichot, Paris

Schaliing, Stockholm

'Schlwartz, Paris

SIMON, Paris

Singier, Strasbourg

WIDLOCHER, Paris

ZARIFIAN, Caen 
FORME ET PRÉSENTATION. Comprimés enrobès (jaunes), gravés: recto: DUPHAR, verso : code d'identification n ${ }^{\circ} 291$, dosés à $50 \mathrm{mg}$. Boile de 30 (présentés sous plaquettes thermoformées). COMPOSITION. Fluvoxamine (maléate) $\mathrm{Cl}$ ou méthoxy-5 [(trifluoro-méthyl)-4 phényl]. luveranine 1 pentanone - 10 - (amino - 2 ethyl) oxime - (E), maleate. Par comp PRO$50 \mathrm{mg}$. Par boîte de $30: 1,5 \mathrm{~g}$. Excipient q.s.p. un comprimé enrobe. PROtement des troubles dépressifs de l'humeur. Son mode d'action est

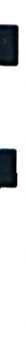

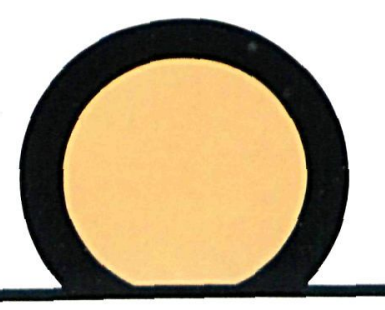

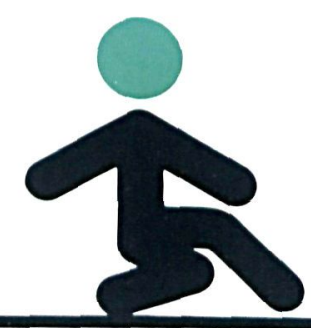

caractèrisé par une inhibition spécifique de la recapture de la sérotonine au niveau des neurones cérébraux tandis qu'il n'interfère pas avec les mécanismes noradrénergiques. Selon les données pharmacologiques, FLOXYFRAL ne présente ni activité stimulante, ni activité sédative et par ailleurs est dénué d'effets anticholinergiques. FLOXYFRAL est sans conséquence sur l'activité psychomotrice. FLOXYFRAL ne présente pas d'effets indésirables au niveau cardio-vasculaire II a été prilise in chez des patients qui présentaient une afloctlon cardiaque sans inconvénient apparent. II ne provoque pas habituellement d'eftet hypotenseur orthostatique. Les eludes do tolerance el deflicacite realisert chez les patients ages nont pas montré de difference par rapport celles conduites dans d autres tranches d'age. La fluvoxamine a monte un effet favorable sur lidee de suicide, observe lors de la premiere semaine de traitement. SORT DU MEDICAMENT. FLOXYFRAL est absorbé rapidement et complètement après son administration orale. FLOXYFRAL est métabolise par le foie et excrété par les reins. Se métabolites primaires sont pharmacologiquement inactifs. PRÉCAU-

TIONS D'EMPLOI. - Les patients avec tendance suicidaire doivent être tout particulièrement surveillés en début de traitement. - L'absorption

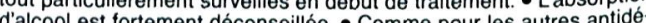
dalcol est torticies an presseurs, a employer avec prudence chez les sujets épileptiques pour esquels Grossesse : bien que ladministration a lanimal nait causé aucune matormation folale, linocuité d'un tel traitement au cours de la grossesse et en période d'allaitementría pas éte élablie. Lutilisation de ce médicament chez la femme enceinte ou en période d'allaitement ne sera décidée qu'après avoir mis en balance les avantages attendus d'une part, et l'éventuel risque thérapeutique d'autre part. VIGILANCE ET CONDUITE. $\bullet$ A fortes doses, ce médicament peut modifier les capacités de réaction des personnes exécutant certaines tâches dange reuses, telles que la manipulation d'appareils ou la conduite d'un véhicule à moteur. INTERACTIONS MEDICAMENTEUSES. - En cas d'administration concomitante de fluvoxamine et d'un médicament contenant du propranolol ou un bêta-bloquant à métabolisme hépatique, il peut être nécessaire de réduire la posologiede ces derniers. II est à noter qu'aucune interaction n'est apparue entre la fluvoxamine nolol - En cas d'administration conpanitant la fluvoxamine ell'atemédicament contenant de la warfarine ou un anti-vitamine Ka méctabo-

lisme hépatique, il peut être nécessaire de réduire la posologie de ces derniers et de surveiller le taux de prothrombine. ll está noter qu'aucune interaction n'est apparue entre la fluvoxamine et la digoxine. D'une façon génerale, l'association de la fluvoxamine à des médicaments métabolisés par le foie ne doit se faire qu'avec prudence til peut être nécessaire de réduire la posologie de ces derniers. INDICATIONS. Etats depressifs de toute nature CONTRE-INDICATIONS. Association aux IMA.O. (il faut respecter un intervalle d'au moins quin. Association aux IMA.O. (1) GARDE. Exceptionnellement une dívation des enzym MISEEN GARDE. Exception du traitement par la fluvoxa enzymos hopatiques est apparue au cours du railome, partois symptobase des données actuelles relatives aux conditions d'utilisation de la fluvoxamine chez l'insuffisant hèpatique, utiliser ce médicament avec précaution chez de tels patients. EFFETS INDESIRABLES. • Phénomènes disparaissant habituellement avec la poursuite du traitement : troubles digestifs (le plus souvent nausees), somnolence, tremblements. Incidents liés à la nature même de la maladie (levée de l'inhibition psychometrice avec risque suicidaire ; inversion trop rapide del'humeur. meur apparition dus

avec un peu d'eau, au milieu ou en fin de repas. La posologie usuelle est de $100 \mathrm{mg}$ par jour (soit 2 comprimés à $50 \mathrm{mg}$ ) en 1 ou 2 prises. Elle sera ajustée en fonction de la réaction du patient et pourra étre portée, si nécessaire, à un maximum de $300 \mathrm{mg}$ par jour. COOT DU TRAITEMENT JOURNALIER. 5.35 francs. SURDOSAGE. II n'y a pas d'antidote spécifique de FLOXYFRAL. En cas de surdosage, l'estomac doit être vidé de que con primés. primes. Une survellance attentive des foncto ns vitas doit étre pratiqueee, ainsi que des thérapeutiques destines asmatiques et pout ains FAL n'est pas tor être élimine par l'emploi d'un salidiuretique ou par dialyse. Lors de l'absorption de doses allant de $0,6 \mathrm{~g}$ (12 comprimes) à $3,5 \mathrm{~g}$ (70 comprimés) de fluvoxamine, aucun retentissement sur lestonctions vilales, en particulier cardio-vasculaires ou neurologiques, n'a éte constate. DUREE DE STABILITE. 3 ans. Tableau A.A.M.M. $n$ T27 2911. Commercia- 


\section{PSYCHIATRIE \& PSYCHOBIOLOGIE PSYCHIATRY \& PSYCHOBIOLOGY}


Rédacteurs en chef: P. BoYer, Paris/Sainte-Anne, J.D. Guelfi, Paris/Sainte-Anne, Y. Lecrubier, Paris/Salpêtrière

\section{Rédacteurs associés}

Psychopathologie

J. ADES, Paris/Colombes

H.S. Akiskal, Memphis, TN

J. DAlery, Lyon

R. Klein-Gittelman, New York

H. Katschnig, Vienne

R. LEVY, Londres

H.G. POPE, Belmont, MA

W. REIN, Tübingen

Diagnostic et classification

N. Mombour, Munich

C.B. Pull, Luxembourg

N. SARtorius, Genève

R.L. SPITZER, New York

Psychobiologie

M. ACKENHEIL, Munich

\section{Comité Scientifique}

N.C. ANDREASEn, Iowa City, IO

M. AsberG, Stockholm

J.L. Ayuso, Madrid

T.A. BAN, Nashville, TN

P. BERNER, Vienne

D. BOBON, Liège

M. Bourgeois, Bordeaux

M. Casas, Barcelone

J. Cottraux, Lyon

R. DANTzer, Bordeaux
P. Baumann, Lausanne

J.-P. Boulenger, Caen

D. Kemalı, Naples

P. LEMOINE, Lyon/Bron

Psychothérapie

A. Braconnier, Paris

S. CONSOli, Paris

P. Jeammet, Paris

C. Perris, Úmea

I. MARKS, Londres

\section{Chimiothérapie}

G. Cassano, Pise

J.A. Costa E Silva, Rio de Janeiro

D. GINESTET, Paris/Villejuif

G. SEDVAll, Stockholm

R. Takahashi, Tokyo +

A. Villeneuve, Beaufort/Québec

H. Dufour, Marseille

A. Feline, Paris/Kremlin-Bicêtre

J. Glowinski, Paris

C. Kordon, Paris

S. Lebovici, Paris

M. Le Moal, Bordeaux

T. Lemperiere, Paris/Colombes

J. H. Loo, Paris

J.J. LOPEZ IBOR, Madrid
Psychopharmacologie clinique

J.M. Danion, Strasbourg

S.Z. LANGer, Paris

A.J. Puech, Paris

T.W. RoBbins, Cambridge, U.K.

Comportement animal et pharmacologie

J. Costentin, Rouen

$\mathrm{Ph}$. Soubrie, Montpellier

Neuropsychologie

M. de BonIs, Paris/Kremlin-Bicêtre

J.A. Gray, Londres

Epidémiologie et Analyse des données

J. Fermanian, Paris

R. von FrENCKELL, Liège

J. LellouCH, Paris/Villejuif

J.P. LÉPINE, Paris

F. Rouillon, Paris/Colombes

(c) 1989 Editions Scientifiques Elsevier, Paris

Tous droits de traduction, d'adaptation et de reproduction par tous procédés réservés pour tous pays.

La loi đu 11 mars 1957, n'autorisant aux termes des alinéas 2 et 3 de l'article 41 , d’une part, que les copies ou reproductions strictement réservées à l'usage privé du copiste et non destinées à une utilisation collective et, d'autre part, que les analyses et courtes citations dans un but d'exemple et d'illustration, " toute représentation ou reproduction intégrale ou partielle, faite sans le consentement de l'auteur ou de ses ayants droit ou ayants cause, est illicite" (alinéa I ${ }^{\text {ef }}$ de l'article 40 ).

Cette représentation ou reproduction, par quelque procédé que ce soit, constituerait donc une contrefaçon sanctionnée par les articles 425 et suivants du Code pénal.

Imprimé en France par l'Imprimeric Saint-Paul, 55000 Bar-le-Duc

Le directeur de la publication: Alain Devanlay Dépốt légal: mars 1989. N $N^{\circ} 1-89-028$

Commission paritaire: 68321
Périodicité: 6 numéros/an 


\section{PSYCHIATRIE}

\& PSYCHOBIOLOGIE

PSYCHIATRY \& PSYCHOBIOLOGY

Volume 4

(1989)

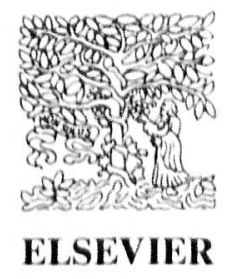

Amsterdam, New York, Oxford, Paris

29, rue Buffon, 75005 PARIS 


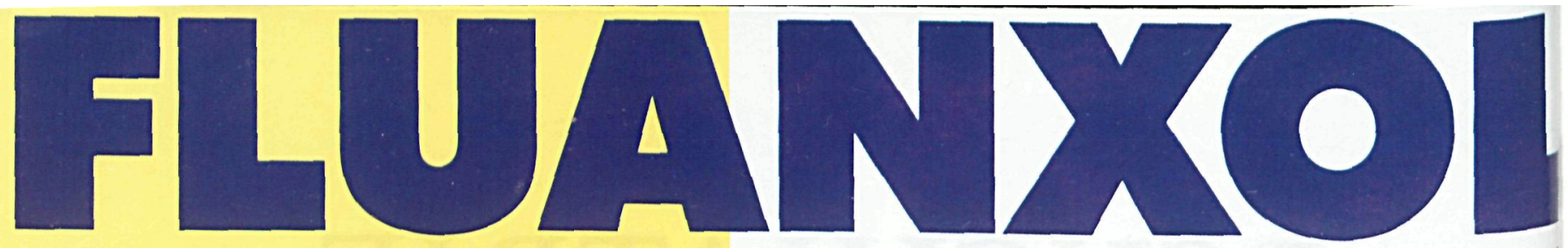

Une gamme

(flupent

neuroleptique

polyvalente

PROPRIETES PHARMACOLOGLUES - Neuroleptique incisif, fomilie des thioxonthenes. SORT DU MEDICAMENT : - FLUAWXOL solution buvable : - concentration serique maximum reinte entre 3 et 8 heures apres l'injection. o temps de $1 / 2$ vie sérique de 24 heures environ ez l'adulie. - FLUAWXOL reterd $2 \%$ of $10 \%$ : e concentration serique maximum ofteinte trre les II' et 17? jours apres l'iniection. - Commun oux deux formes: $\bullet$ le flupentixol est degrode

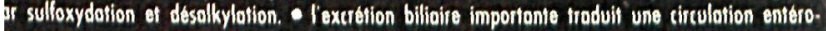
spatique intense. - l'alimination feccale est cing lois plus importante que lélimination urinaire. RESENTATIONS : - FLUANXOL solution buvable a $4 \%$ (illupentixol dichlorhydrate) Hacon mple-gouties de $10 \mathrm{ml}$ - FLUANXOL retard $2 \%$ (ilupentixol deconoote) $(20 \mathrm{mg} / \mathrm{ml}$ ). Solute lectable L.M. a action prolonget. Boire de 4 ampoules de $1 \mathrm{ml}$ - FLUAWXOL retard $10 \%$ (llupentixol canoate) $100 \mathrm{mg} / \mathrm{ml}$ ). Solute injectable I.M. a action prolangée. Boite de 1 ampoule de $1 \mathrm{ml}$. DDICATIONS : Syndromes psychotiques aigus ou chroniques. CONTRE.INDICATIONS : ^ Risque de glau. me par fermeture de l'angle. - Risque de rétention urinaire liee a des troubles uratiro-prosiatiques. intre-indications, associations deconseillees : alcool, levodopo. MISE EN GARDE : Syndrome malin. Tout aitement neuroleptique doit etre suspendu en cas de survenue d'une hyperthermie, celle.ci pouvont re un des elements du syndrome malin (polleur, hyperthermie, troubles vegetarils) decrit avec les uroleptiques: EFFETS INDESIRABLES ET INTERACTIONS: (eux de la plupart des neuroleptiques: fets neurologiques, vegetratils, endocriniens of métaboliques. Se reporter oux monographies figurant ans le dictionnaire des specialites pharmaceutiques. PREECAUTIONS D'EMPLOI: • l'absorption de boissons coolisees est fortement deconseillee pendant le rroitement. - Surveillance rentorcee shez les epilepriques ossibilitie d'aboissement du seuil eppileptogène). • Urilisation ovec prudence chez les parkinsoniens néces. tant un traitement neuroleptique. PRUDENCE : • Chez les sujets ages, en raison de leur importante sensibilitie edation et hypotension). - Dons les affections cardio-vasculaires graves, en raison des modifications emodynamiques, en particulier l'hypotension. $\bullet$ Dans les insuffisances renale et hepatique, en roison

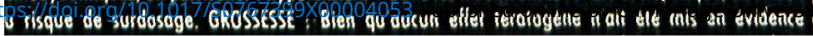

aves prudence ef sous surveillone stricte ther to femme enteins SATION OE LA FORME INJECTABLE RETARD : Avant d'instituer un traile. FLUANXOL retard, il est utile de tester la sensibilite du poli le FLUANXOL solute buvable. MODE DEEMPLOI ET POSO - FLUANXOL solution burable : Posologie strictement indivic Conction du tableau clinique : $\bullet$ elfier antiasychatique ef desinhtibiter $80 \mathrm{mg} /$ jour en une ou deux prises. $\bullet$ effet antidelirant et anti-halluc 80 o $200 \mathrm{mg}$ /iour, éventuellement jusqu'o $400 \mathrm{mg}$ y jour en milieu ho - FLUANXOL retard : Voie I.M. prolonde. Le plus souvent utilise du troitement neuroleptique par voie orale. Chez l'adulie : p strictement individuelle en tonction du tableau clinique. • effet antips el désinhibiteur : de 20 a $80 \mathrm{mg}$ toules les 2 a 3 semaines. e effer an at anti-hallucinatoire : de 80 o $300 \mathrm{mg}$ toutes les 2 o 3 semain LES SUJEIS AGES, LES EPILEPIOUUES : posologie reduire o lo moitie ou de la posologie mentionnèe ci-dessus.SURDOSAGE : - Syndrome park gravissime, coma. - Traitement symptomatique en milieu specialise. TA FLUANXOL solution buvable mis sur le marche en 1975. A.M.M. : 3 FLUANXOL retard $2 \%$; A.M.M. 318.096.5. Amp. I mL. Mis sur le marche FLUANXOL retord $10 \%$ : A.M.M. : 326.349.6. Amp. I ml. Mis sur le m. 1985. PRIX: FLUANXOL sol, bUY. : $50,90 \mathrm{~F}+$ S. H.P. fllocon de $10 \mathrm{ml}$. FLU retard $2 \%$ amp. $1 \mathrm{ml}: 57,70 \mathrm{~F}+5$. H.P. boite de 4 , FUUANXOL reto amp. I ml : 58.90 F + S.H.P. (boite unitoire). Remb. Sect: Soc. o 70

7 Loboratoires CLIN MIDY 20, rue des Fosses Si. Jocques 75240 PARIS CEDEX 05 


\section{PSYCHIATRIE \& PSYCHOBIOLOGIE PSYCHIATRY \& PSYCHOBIOLOGY}

\section{OBJECTIFS}

L'objectif de Psychiatrie \& Psychobiologie est de publier les résultats de travaux originaux dans les domaines qui intéressent, à l'heure actuelle, la Psychiatrie tels que: psychopathologie, nosographie, chimiothérapie, psychothérapie, méthodologie des essais cliniques, perturbations biologiques et pathologie mentale, psychophysiologie, neuropsychologie, comportement animal.

Cet objectif large mais centré sur la publication d'articles originaux est destiné à:

1) faciliter la circulation des idées et des travaux au sein des pays européens,

2) permettre une meilleure connaissance mutuelle des évolutions survenant dans la communauté psychiatrique internationale.

Les communications brèves seront publiées dans des délais rapides afin de rendre le plus actuel possible les informations présentées dans cette revue.

\section{AIMS AND SCOPE}

Psychiatry \& Psychobiology presents the results of original research relative to those domains which are presently of interest to psychiatry: psychopathology, nosography, chemotherapy, psychotherapy, clinical methodology, biological disorders and mental pathology, psychophysiology, neuropsychology, as well as animal behavior.

This large scope, emphasizing, nevertheless, the publication of original articles, is meant to:

1) encourage the exchange of ideas and research within Europe,

2) enable, within the international psychiatric community, an improved level of scientific communication.

Short communications will be published rapidly in order to keep informations in this journal up-to-date.

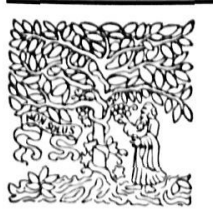

\section{EDITIONS SCIENTIFIQUES ELSEVIER}

29 , rue Buffon, F-75005 Paris

Tél. : (1) 47.07.11.22 - Télex: 202400 F - Télécopie: (1) 43.36.80.93

ABONNEMENTS/SUBSCRIPTIONS 1989 (Vol. 4)

Un an/annual subscription; 6 numéros/6 issues: FF 450 (France), FF 500 Autres pays (other countries).

Adresser ordre et paiement à /Address order and payment to: Editions Scientifiques Elsevier, 29 rue Buffon, 75005 Paris, France.

Banque/Bank: CCP Paris, No $1904540 \mathrm{H}$.

- Les abonnements sont mis en service dans un délai de 4 semaines après réception du paiement. Ils partent du premier numéro de l'année/Subscriptions begin 4 weeks after receipt of payment and start with the first issue of the calendar year.

- Numéros de l'année et volumes antérieurs doivent être commandés à l'éditeur/Back issues and volumes should be ordered from the publisher. - Les réclamations pour les numéros non reçus doivent parvenir dans un délai maximal de 3 mois après la parution/Claims for missing issues should be made within three months of publication.

- Les revues sont expédiées par avion, sans supplément, dans les pays suivants: D.O.M.-T.O.M., Bénin, Burkina-Faso, Cameroun, Comores, Congo, Côte-d'Ivoire, Djibouti, Gabon, Guinée, Madagascar, Mali, Mauritanie, Niger, République Centrafricaine, Sénégal, Tchad, Togo, Tunisie/Journals are sent by SAL air delivery (Surface Airlifted Mail) to the following countries: South Africa, Argentina, Australia, Brazil, Canada, PR China, Hong Kong, India, Israel, Japan, South Korea, Malaysia, Mexico, Pakistan, Singapore, Taiwan, Thailand, USA, New Zealand.

Tarifs aériens pour d'autres pays sur demande/Airmail rates for other countries are available upon request.

PUBLICITE/ADVERTISING: Editions Scientifiques Elsevier (P. Richard).

(C) 1989 Editions Scientifiques Elsevier, Paris

Tous droits de traduction, d'adaptation et de reproduction par tous procédés réservés pour tous pays.

La loi du 11 mars 1957, n’autorisant aux termes des alinéas 2 et 3 de l'article 41 , d’une part, que les copies ou reproductions strictement réservées à l'usage privé du copiste et non destinées à une utilisation collective et, d'autre part, que les analyses et courtes citations dans un but d'exemple et d'ilhustration, " toute représentation ou reproduction intégrale ou partielle, faite sans le consentement de l'auteur ou de ses ayants droit ou ayants cause, est illicite " (alinéa I ${ }^{\mathrm{ct}}$ de l'article 40 ).

Cette représentation ou reproduction, par quelque procédé que ce soit, constituerait done une contrefaçon sanctionnée par les articles 425 et suivants du Code pénal. 


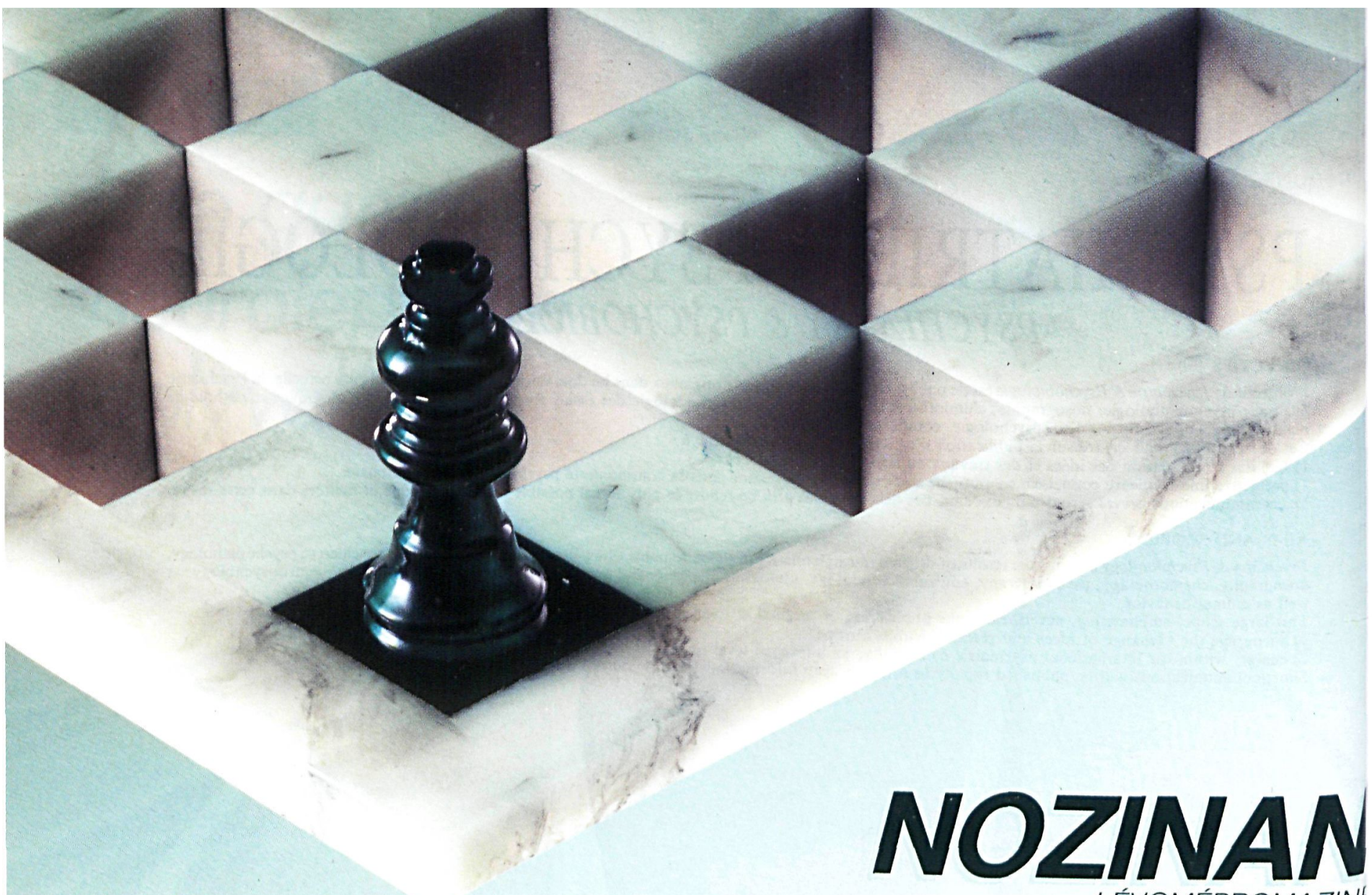

LÉVOMÉPROMAZINE

\section{De l'anxiété courante à l'angoisse psychotique}

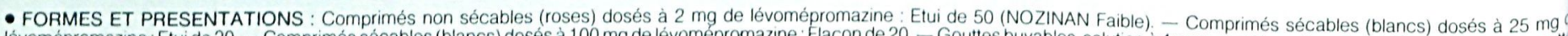

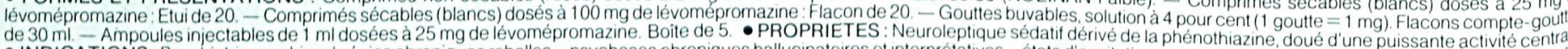

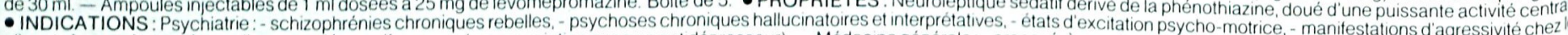

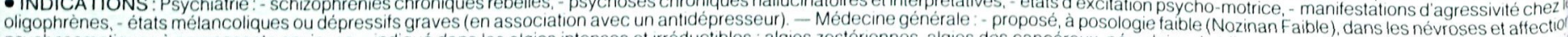

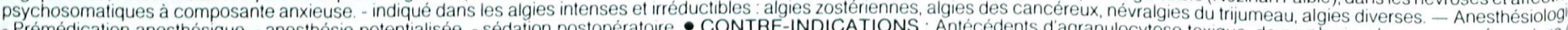

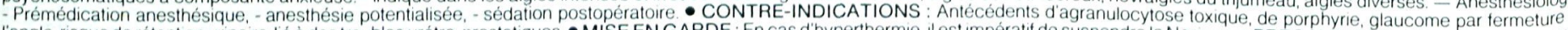

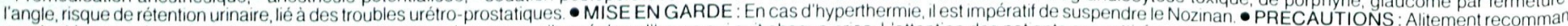

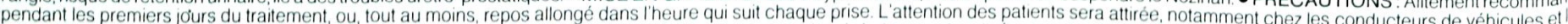

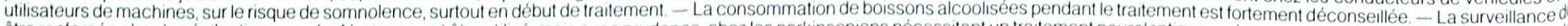

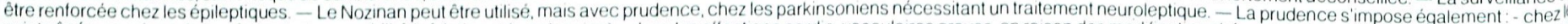

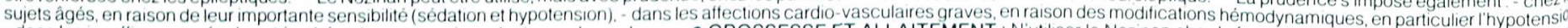

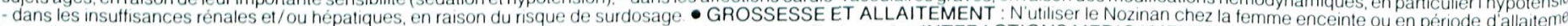

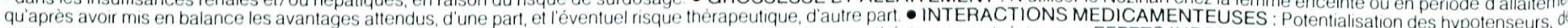

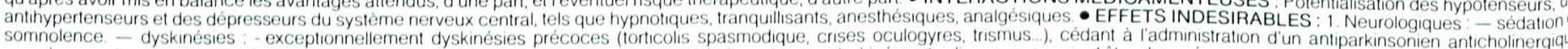

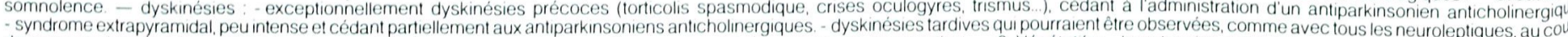

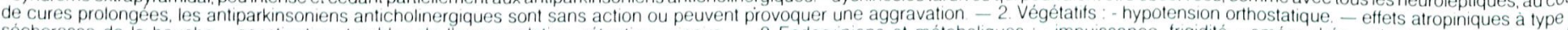

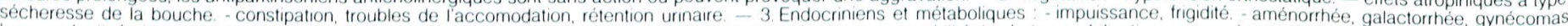

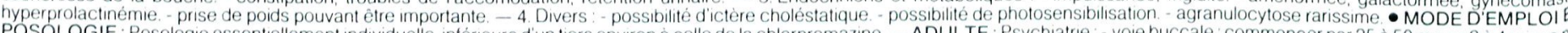

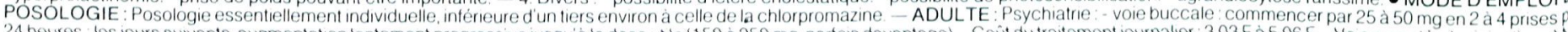

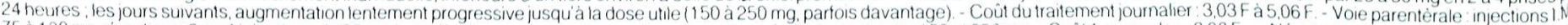

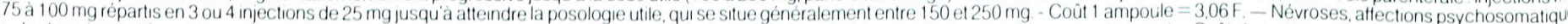

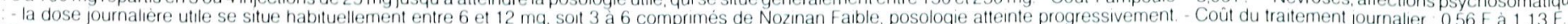

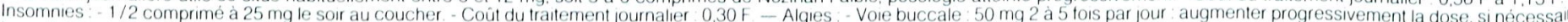

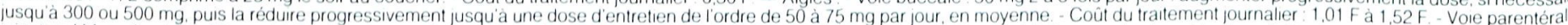

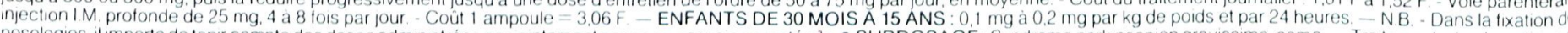

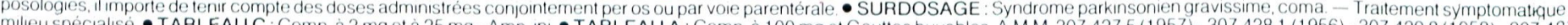

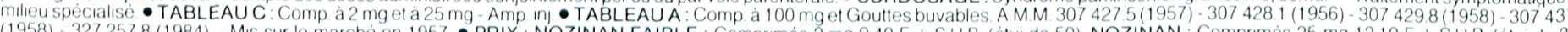

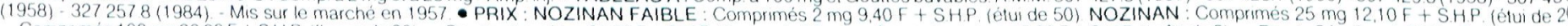

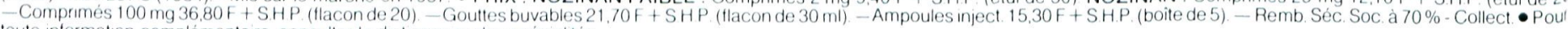
toute information complémentaire consulter le dictionnarre des spécialités

\section{SPECIA}

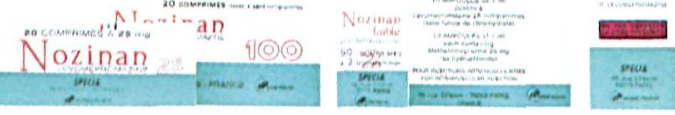

16, rue Clisson - 75636 PARIS CEDEX 13 - Tél. : (1) 4584.11 .3 
Anxiety-related inhibition

C.B. Pull and D. Widlöcher (Luxembourg and Paris, France) $\ldots \ldots \ldots \ldots \ldots \ldots \ldots \ldots$

Utilisation du DSM III en psychiatrie de l'enfant et de l'adolescent

M.F. Le Heuzey, O. Halfon, N. Crepin, P. Betolaud et M. Dugas (Paris, France) ..........

Patterns of care in Italian psychiatric services and psychosocial outcome of schizophrenic patients.

A three-year prospective study

D. Kemali, M. Maj, B. Carpiniello, R.D. Giurazza, M. Impagnatiello, D. Lojacono, P. Martini and G. Morandini (Naples, Cagliari, Trieste, Cosenza, Arezzo and Verona, Italy) . . . . . ....

La perception du préadolescent. Essai de définition d'un indicateur de santé positive: l'intégration sociofamiliale

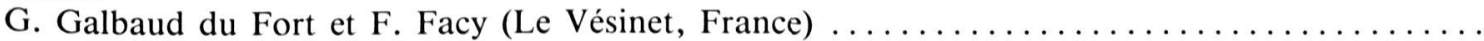

Monitoring of tricyclic antidepressant plasma levels and clinical response: a review of the literature. Part I.

I.R. de Oliveira, P.A.S. Do Prado-Lima and B. Samuel-Lajeunesse (Bahia, Brazil and Paris,

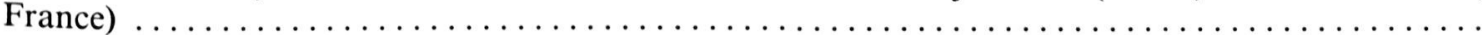

Utilisation conjointe de la méthode du cotateur aveugle avec temps aveugle et de la procédure de minimisation dans un essai comparatif de la viloxazine IV versus clomipramine IV avec contrôle des taux plasmatiques

D. Leguay, D. Robert, A. Turcant, J.B. Garre, J.P. Lhuillier, J. Lavoisy, J.M. Marion, P. Allain

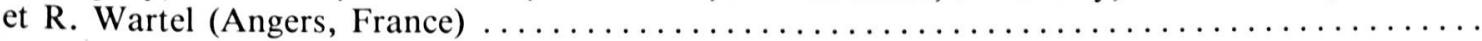

La psychiatrie et la crise morale

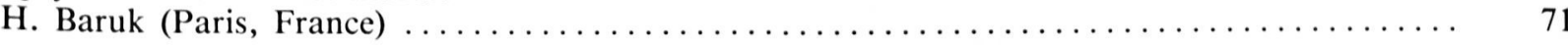

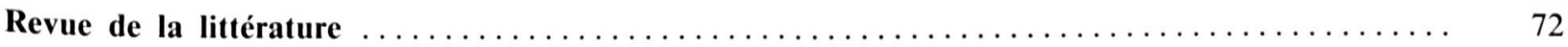

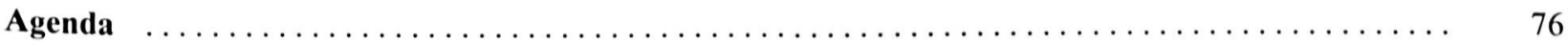

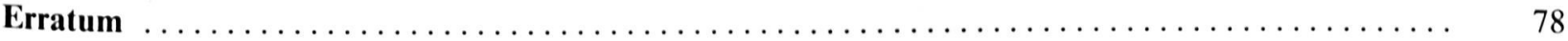

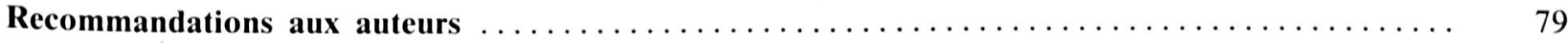

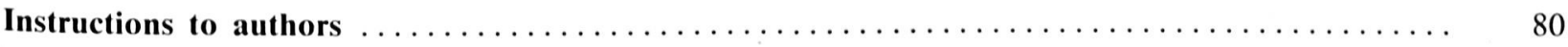

Cité dans: Biological Abstracts; CNRS/Pascal; Excerpta Medica; Psychological Abstracts 

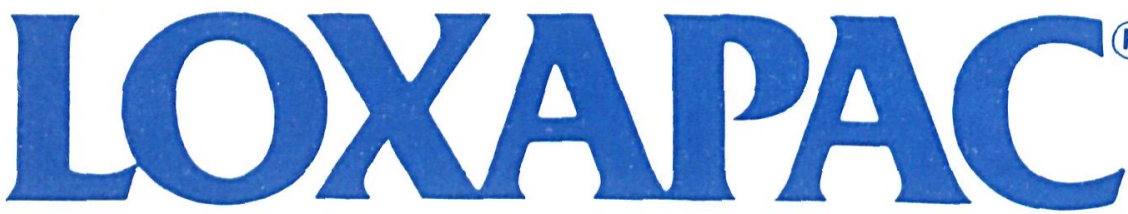

loxapine traiter

\section{la désorganisation psychotique}

$(B)$

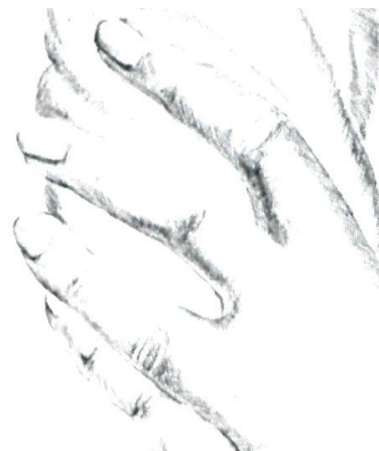

Formes et présentations : - Comprimés à $50 \mathrm{mg}$ non sécables (bleus) : boite de 12. • Comprimes a $25 \mathrm{mg}$ non sécables (blancs) : boite de 30 . Solution buvable: flacon de $30 \mathrm{ml}$ Composition : - LOXAPAC 50 comprimés : Ioxapine (DCl) sous forme de succinate, $50 \mathrm{mg}$ par unité, $600 \mathrm{mg} /$ boite - LOXAPAC 25 comprimés : loxapine (DCl) sous forme de Succinate, $25 \mathrm{mg}$ par unite, $70 \mathrm{mg}$ par boite - LOX flation Buva me rapidement flacon. Sort du medicament : La loxapine est rapidement absorbée. Le pic plasmatique est atteint environ $1 \mathrm{~h} 30$ après la prise du produit. Après résorption digestive, il existe lor d premier passage, un captage hépatique qui la transforme rapidement pour les $2 / 3$ en métabolites hydroxylés en 7 et 8 possédant les mêmes propriétés ainsi qu'en déméthylloxapine, elle-même hydroxylée en 7 et 8 . Sa demi-vie biologique est de l'ordre de 8 heures et son élimination se fait à $70 \%$ par voie rénale, essentiellement sous forme de métabolites coniugués Proprités. Neuroleptique La loxapine, principe actif du OXAPAC est le chef de file d'une nouvelle famille. les dibenzoxazépines Grâce à ses propriétés antipsychotiques, I réduit les hallucinations et le délire. antipsychotiques, Il reduit les hallucinations et le delie. améliorant la cohérence de la pensée. Indications : Psychoses chroniques (délires systématisés chroniques et
schizophrénies). - Etats maniaques et hypomaniaques. États schizophrénies). - Etats maniaques et hypomaniaques. - Etats d'agitation, d'agressivité et anxiété associée à des troubles psychotiques ou psychopatiques, à la débilité mentale, à l'alcoolisme, à la comitialité. - Tratement lors du sevrage des toxicomanes. Contre-indications : - Comas ou états graves causés par l'alcool, certains médicaments barbituriques Hypersensibilité connue aux médicaments de cette classe. Mise en garde, précautions d'emploi, effets indésirables voir la monographie du dictionnaire Vidal Mode d'emploi et posologie : Réservé à l'adulte. La bioéquivalence des formes solution buva Cele-ci sera do próten comprones ou la solution buvable. Cello-ci sera doppeterence diluée dans un seume sicre ou un jus drar seule prise le soir ou fractionnée au cours de la journée. La posologie est ajustée en fonction de la maladie. progressivement au départ. puIs maintenue géneralement entre 200 et $300 \mathrm{mg}$ par jour. Elle pourra s'élever à $600 \mathrm{mg}$ par jour dans certains cas mais le plus souvent ne depassera pas $300 \mathrm{mg}$ par jour. Coût du traitement journalier moyen $11,50 \mathrm{~F}$. Surdosage : II n'existe pas de signe spécifique du surdosage par Loxapac. Aucun antidote spécifique connu: seul un traitement symptomatique sera entrepris. Tableau A. A.M.M. 322277.0 (1978) comp à 25 mg A.M.M. 322275.8 (1978) comp à $50 \mathrm{mg}$ AMM 3233420 (1980) sol buv Mis sur le marché en 1980 Prix: LOXAPAC 50:38.00 F + SHP (12 comprimés) LOXAPAC $25: 47.00 \mathrm{~F}+5 . \mathrm{HP}$ (30 comprimes) de $30 \mathrm{ml}$. Remb. Séc. Soc à $70 \%$ - Collect

\section{Edenk}

\section{Laboratoires LEDERLE}

Information Médicale 74, rue d'Arcueil - Immeuble léna 94578 RUNGIS Cedex - Tél. (1) 46.87.23.19

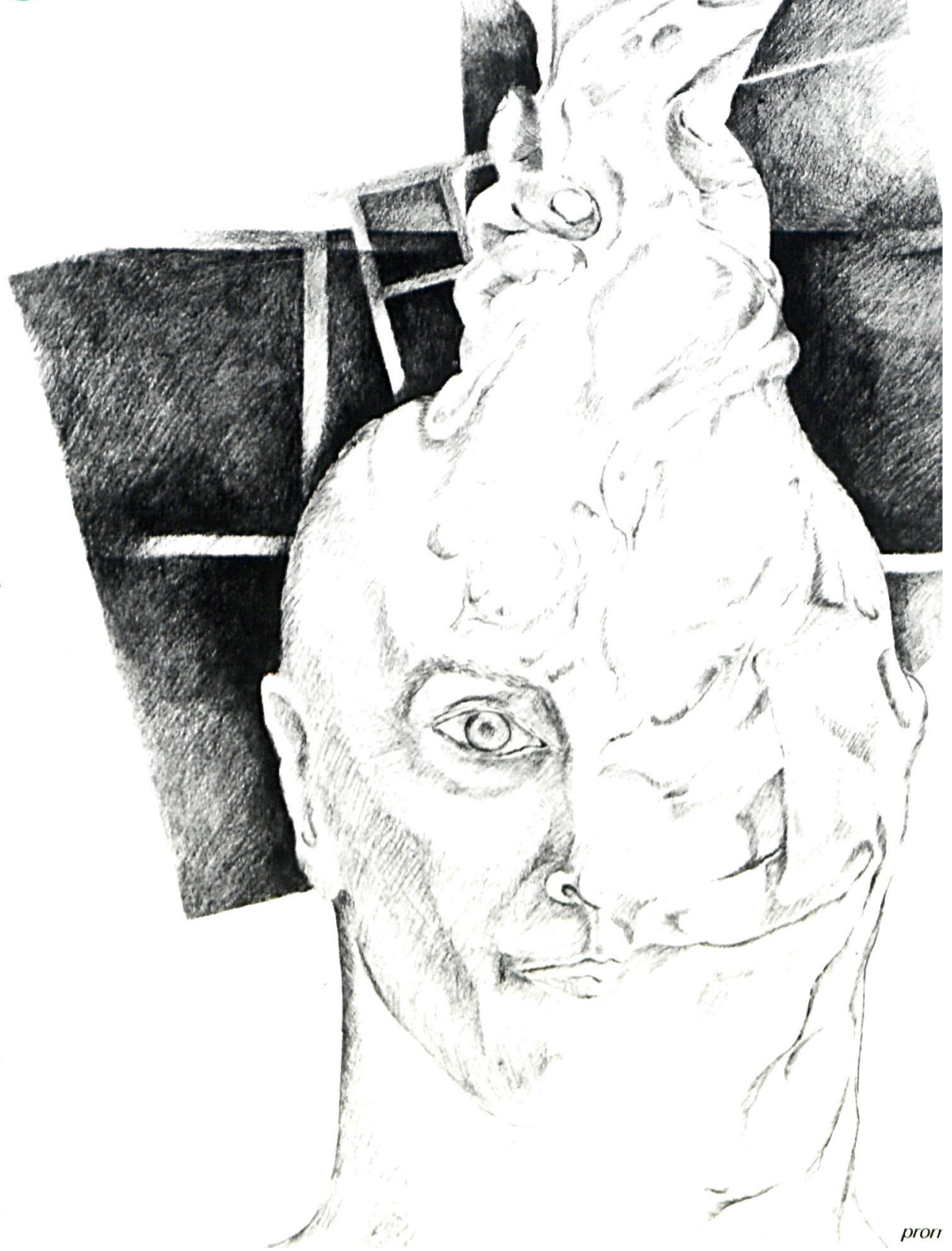

